\section{Exotic astrophysics}

\section{G. Edmunds}

Black Holes, White Dwarfs, and Neutron Stars: The Physics of Compact Objects.

By Stuart L. Shapiro and Saul A. Teukolsky.

Wiley: 1983. Pp.645. Hbk £42.70, \$59.80; $p b k £ 20.45, \$ 28.70$.

WHEN they die, stars leave very interesting corpses. Depending on their mass, single stars either settle down to fade away as dense white dwarfs supported by quantum mechanical degeneracy pressure, or explode as spectacular supernovae. These explosions may sometimes disrupt the star completely, but the compression of matter to very high densities in the cores of the exploding stars frequently produces either a neutron star or, if the core mass is too large, collapse to a black hole.

The dense stellar remnants may also be left in a binary system of stars in which the other member is still undergoing its normal evolution. The exchange of matter from the living star onto the dead can result in a spectacular life-after-death, particularly in the emission of X-rays. A major theoretical effort to understand the structure and properties of compact remnant stars was stimulated during the 1960 s and 1970 s by two observational developments; one was the launch of rockets and satellites which detected the X-ray sources, the other was the discovery of pulsars.

This impressive volume by Shapiro and Teukolsky, both of whom have been intimately involved with the field, is aimed at introducing the physics of compact objects to advanced undergraduates and first-year graduate students. The wide reach of physics involved - ranging through general relativity, thermodynamics and nuclear, particle, solid-state and plasma physics - inevitably means that much of the underlying theory has to be taken on trust. For example, derivation of astrophysical results reasonably starts by assuming expressions for the Schwarzchild metric and Weinberg-Salam-Glashow weak interaction theory. Likewise a summary of basic astronomy is provided as an appendix, but it is short and again the book will be more understandable to those with some previous knowledge.

Many physical mechanisms in compact objects are still uncertain. We do not know exactly why supernovae explode, although the most likely explanation seems to be simple pressure generated by a shock wave that passes out from the core as it "bounces" back from gravitational collapse when its equation of state stiffens at high densities. The mechanism that generates the intense radiation from pulsars is even more obscure. The authors are commendably brief and non-committal on topics of this kind where not much has been reliably established. Two important areas of recent research that are well covered are accretion disks - the region from which much of the observed radiation originates as material loses angular momentum and spirals in towards the compact object and the generation of gravitational waves.

The book is well produced and has many interesting exercises which I found to be full of ideas for use in setting this year's exam questions. My own students beware! It is an excellent introduction to the current state of research - references reach right up to 1982 - that will be of value not only to students but to any physicist or astronomer who does not work directly on the problems of these exotic objects.

M.G. Edmunds is a Lecturer in the Department of Applied Mathematics and Astronomy at University College, Cardiff.

\section{Making waves}

\section{H. Lipson}

\section{The Physics of Vibrations and Waves,} 3rd Edn.

By H. J. Pain.

Wiley: 1983. Pp.416. Hbk £18.95, \$35; pbk $17.50, \$ 13.90$.

Vibrations and Waves.

By W. Gough, J. P. G. Richards

and R. P. Williams.

Ellis Horwood: 1983. Pp.278.

Hbk £27.50, \$64.95; pbk £7.50.

Waves and Photons: An Introduction to Quantum Optics.

By Edwin Goldin.

Wiley: 1983. Pp.211. £22.95, \$33.55.

Concepts of Quantum Optics.

By P. L. Knight and L. Allen.

Pergamon: 1983. Pp.217. Hbk f16.75, $\$ 30 ; p b k £ 8.95, \$ 16$.

SOME time ago it was being suggested that physics should not be taught in sections heat, light and so on - but as a single entity. Topics should be general, coming down to earth, as it were, when obvious applications appared. Vibrations and waves was mentioned as an appropriate subject for such an approach, but when asked for other examples the proponents of the idea could not think of any. So it is that physics teaching continues very much in its standard form.

If the idea had worked, these four books would have served well to illustrate the argument. The first one, the new third edition of Pain's The Physics of Vibrations and Waves, is the most elementary. It begins with mechanical systems, which are the easiest for students to envisage, and continues with electric transmission lines, electromagnetic waves, optical waves and finally wave mechanics. Fourier analysis and Fourier transforms are also introduced, and the book contains many wellthought-out problems.
Some of the ideas are, perhaps, oversimplified, however. For example Fermat's principle is stated correctly, but is treated purely as that of least time. Nor is the basis of the principle explained. But my main criticism is that some of the diagrams are inexcusably poor; many of the sine curves are not accurately sinusoidal, and the shapes of parabolic, elliptical and Gaussian curves are unsatisfactory.

Of Gough, Richards and Williams's Vibrations and Waves I have no such complaint. It is pitched at about the same level as Pain's book, but is shorter and concentrates on sound and light, giving at one point a detailed discussion of the perception of musical sounds (here it is surprising, given the book's provenance, that no mention is made of the part played by starting transients). Again, there are some excellent problems for the students to test their understanding. Finally, a computer program for the visual presentation of waves is provided. Like Pain, however, these authors devote little space to double refraction in crystals.

The third book, Waves and Photons, is less general and more ambitious than the first two, and would certainly not be suitable as an introductory textbook. Like the other authors, Goldin starts off with waves in general but then concentrates on light; he also includes some thoughtprovoking problems. But the main objective, as stated on the book's jacket, is to try to remove the paradox between wave and quantum optics. The writing is stimulating and intellectually demanding, but it is not easy to say whether the work succeeds; to the experimental physicist like myself, the answer is probably "no", but those who put their faith in mathematics may be satisfied.

The final book, Knight and Allen's Concepts of Quantum Optics is quite different again; it would be appreciated by only the very brightest students. The book contains six short chapters, all theoretical, on topics that the authors consider to be of fundamental importance, and these are followed by reproductions of 17 original papers to illustrate points made in the earlier part of the book.

Paper 1 is the first published paper of G. I. Taylor (1909), describing his efforts to see if very weak light could give Young's fringes, even if the probability that two photons could simultaneously pass through the two slits was infinitesimal. (The longest exposure was three months!) J. J. Thomson suggested the experiments, and the fact that perfectly clear fringes were found surprised everyone. It is amusing, in a way, that such a simple experiment as Young's should inspire so much advanced thinking, including the typically Diracian statement that a photon can interfere only with itself. This conclusion, if it can be called such, seems to be negated by Paper 7, by Magyar and Mandel, in which they describe interference fringes obtained from two separate 